\title{
Aspects extrapulmonaires des exacerbations de bronchopneumopathie chronique obstructive
}

\author{
Extra-Pulmonary Aspects of Exacerbations of Chronic Obstructive Pulmonary Disease (COPD)
}

\author{
D. Viglino $\cdot$ M. Maignan
}

Reçu le 28 février 2019; accepté le 26 juin 2019

(C) SFMU et Lavoisier SAS 2019

Résumé La bronchopneumopathie chronique obstructive (BPCO) est une maladie systémique aux multiples atteintes, émaillée d'exacerbations. L'inflammation, l'hypoxémie, les troubles de la coagulation et les perturbations des interactions cœur-poumon expliquent en partie les atteintes non pulmonaires observées notamment lors d'exacerbations aiguës. Les événements cardiovasculaires sont la première cause de mortalité des patients BPCO, et leur recrudescence est observée pendant plusieurs semaines après une exacerbation. Aux urgences, la prise en charge des patients en exacerbation de BPCO repose donc en plus du support ventilatoire sur une bonne évaluation des potentielles pathologies associées telles que l'insuffisance cardiaque, l'ischémie myocardique, l'insuffisance rénale ou encore la maladie thromboembolique. Cette évaluation globale permet d'adapter les thérapeutiques parfois délétères sur la fonction cardiaque ou l'équilibre acide-base et de prendre en compte le risque extrapulmonaire dans le choix du parcours de soins du patient. Dans cette mise au point, nous abordons quelques explications physiologiques des multiples perturbations observées au cours de l'exacerbation de BPCO, et proposons une vision globale de l'évaluation de ces patients admis pour une dyspnée ou un autre motif pouvant être en rapport avec la BPCO.

Mots clés Bronchopneumopathie chronique obstructive Exacerbation · Comorbidités · Urgences

\begin{abstract}
Chronic obstructive pulmonary disease (COPD) is a systemic disease with multiple comorbidities and organ disorders, punctuated by exacerbations. Inflammation,
\end{abstract}

\section{Viglino $\cdot$ M. Maignan $(\bowtie)$}

Laboratoire HP2 (Hypoxia Pathopysiologies,

Inserm U 1042), université de Grenoble-Alpes,

Saint-Martin-d'Hères, 69, avenue Centrale,

F-38400 Grenoble, France

e-mail : mmaignan@chu-grenoble.fr

Urgences Samu-Smur, CHU de Grenoble-Alpes,

CS 10217, F-38043 Grenoble cedex 09, France hypoxemia, coagulation disorders and disturbances of the heart-lung interactions explain in part the non-pulmonary disorders observed especially during acute exacerbations. Cardiovascular events are the leading cause of death in COPD patients, and their recrudescence is observed for several weeks after an exacerbation. The management of these patients in emergency departments consists therefore, in addition to the ventilatory support, in a good evaluation of concomitant heart failure, myocardial ischemic signs, renal failure, or thromboembolic disease. This global evaluation makes it possible to adapt the sometimes deleterious therapies on the cardiac function or the acid-base balance, and to take into account the extra-pulmonary risk in the choice of the patient's care pathway. In this review, we discuss physiological explanations of the multiple disturbances observed during the exacerbation of COPD, and provide a global vision of what should be included in any evaluation of these patients admitted for dyspnea or any other reason that may be related to COPD.

Keywords Chronic obstructive pulmonary disease · Exacerbation - Comorbidities - Emergency care

\section{Introduction}

Contrastant avec sa définition purement pulmonaire, la bronchopneumopathie chronique obstructive (BPCO) est en réalité une maladie aux atteintes multiples, "systémiques » [1-4], s'exprimant sous la forme de nombreux phénotypes [5] (Tableau 1). La physiopathologie des atteintes extrapulmonaires de la BPCO repose essentiellement sur des lésions systémiques induites par l'inflammation et l'hypoxémie chronique ou intermittente. Les malades BPCO présentent également de nombreux désordres métaboliques liés au tabagisme, à la sédentarité ou encore à certains traitements, autant de facteurs intermédiaires favorisant l'émergence de comorbidités. 


\begin{tabular}{|ll|}
\hline $\begin{array}{l}\text { Tableau } 1 \text { Listes des pathologies extrapulmonaires associées } \\
\text { aux exacerbations de bronchopneumopathie chronique } \\
\text { obstructive }\end{array}$ \\
\hline $\begin{array}{l}\text { Événements } \\
\text { cardiovasculaires } \\
\text { majeurs }\end{array}$ & $\begin{array}{l}\text { Infarctus du myocarde } \\
\text { Insuffisance cardiaque }\end{array}$ \\
Futres & Fibrillation atriale \\
& Accident vasculaire cérébral \\
& Stéatose hépatique \\
& Syndrome d'apnée du sommeil \\
& Maladie thromboembolique \\
& Diabète \\
& Insuffisance rénale \\
& Douleur \\
& Anxiété et syndrome dépressif \\
& Ulcère gastroduodénal \\
\end{tabular}

La compréhension des aspects extrapulmonaires de la BPCO est cruciale. Ces aspects extrapulmonaires sont responsables en grande partie de la morbimortalité et des durées de séjour observées lors des hospitalisations [6,7]. Notamment, l'insuffisance cardiaque, les troubles du rythme cardiaque, le diabète, l'accident vasculaire cérébral et l'embolie pulmonaire sont tous individuellement associés à une augmentation des durées de séjour hospitalier, des coûts et de la mortalité des exacerbations de BPCO [7,8]. Les maladies cardiovasculaires sont la première cause de mortalité des patients BPCO [3]. La sévérité des atteintes extrapulmonaires des patients BPCO est variable selon les individus. Plusieurs phénotypes de patients peuvent être ainsi individualisés : par exemple, les patients dits « exacerbateurs fréquents » dont la morbidité est surtout pulmonaire ; les patients au profil plus extrapulmonaire avec une masse graisseuse importante, une inflammation systémique accrue et de nombreuses comorbidités cardiovasculaires $[9,10]$.

L'exacerbation de BPCO est un événement marquant de l'évolution de la maladie pulmonaire chronique, mais aussi des aspects extrapulmonaires de la BPCO. Les modifications des régimes de pression thoracique, de l'hématose ou encore du système de la coagulation (activation plaquettaire [11] et thrombocytose [12]) ont certes un impact sur la physiologie pulmonaire mais aussi sur le cœur, les vaisseaux, etc. La compréhension de l'exacerbation de BPCO doit donc intégrer ces aspects extrapulmonaires afin d'envisager une démarche médicale holistique qui sera bénéfique au patient.

L'objectif de cette mise au point est de décrire certains aspects extrapulmonaires jouant un rôle lors des exacerbations de BPCO afin d'illustrer l'importance d'une compréhension globale de la maladie BPCO. Toutes les comorbidités étroitement associées à la BPCO et aux exacerbations ne seront pas abordées. Notre but est seulement de montrer que la BPCO doit être comprise comme une maladie systémique.

\section{Insuffisance cardiaque}

La prévalence d'une insuffisance cardiaque chez les patients BPCO en phase stable est d'environ $20 \%$ [13]. Elle augmente chez les patients BPCO âgés, et elle est corrélée à une surmortalité [13-15]. La présence d'anomalies reflétant un dysfonctionnement cardiaque, telles que les arythmies ventriculaires et la fibrillation auriculaire, a été identifiée comme doublant le risque de mortalité des patients BPCO [16]. De même, la présence d'œdème pulmonaire lors des exacerbations de BPCO est associée à une mortalité accrue [17]. Une élévation des NT-pro-BNP est un marqueur indépendant de la mortalité des exacerbations de BPCO [18]. Les patients BPCO présentant une insuffisance cardiaque sont également plus souvent hospitalisés et ont un risque accru de présenter une exacerbation sévère $[19,20]$.

La relation entre emphysème pulmonaire et insuffisance cardiaque est décrite depuis les années 1950 [21, et de nombreux travaux ont depuis illustré la physiopathologie croisée entre emphysème, BPCO et insuffisance cardiaque $[3,22]$. Plusieurs mécanismes physiopathologiques pulmonaires concourent à l'instauration d'une insuffisance cardiaque : l'hypertension artérielle pulmonaire induite par le remodelage vasculaire pulmonaire posthypoxique et inflammatoire [23] ; l'hyperinflation pulmonaire et les pressions téléexpiratoires positives qui diminuent le retour veineux et modifient la cinétique du ventricule droit ; l'hypoxie tissulaire qui provoque la production de radicaux libres et une dysfonction endothéliale $[3,22,24]$. Parallèlement, l'insuffisance cardiaque majore les troubles ventilatoires du patient BPCO du fait de la congestion pulmonaire (épaississement des parois pulmonaires et perte d'élasticité), de la dilatation du ventricule droit (compression pulmonaire) et d'une modification des rapports ventilation/ perfusion (inhomogénéité de la ventilation qui s'ajoute à l'inhomogénéité de la ventilation du patient BPCO) [22].

Lors d'une exacerbation de BPCO, ces synergies physiopathologiques jouent un rôle primordial. Notamment, l'hyperinflation dynamique pulmonaire aggrave la défaillance ventriculaire droite [3]. Les thérapeutiques utilisées à visée pulmonaire peuvent également avoir un impact négatif sur la fonction cardiaque. Ainsi, la ventilation non invasive exerce une pression extrinsèque sur le ventricule droit et diminue le retour veineux intrathoracique, même si elle aura un effet bénéfique global en cas d'insuffisance ventriculaire gauche. L'administration de bronchodilatateurs d'action rapide (salbutamol et/ou ipratropium par exemple) peut aggraver la dysfonction myocardique tant par l'effet tachycardisant que par l'augmentation de la consommation en oxygène du myocarde [25]. Enfin, l'administration de furosémide rendue parfois nécessaire par la présence d'une congestion pulmonaire pourrait modifier l'équilibre acidobasique du patient et favoriser une hypoventilation [26,27]. Lorsque la phase aiguë de 
l'exacerbation est terminée, la persistance d'une insuffisance cardiaque pourra limiter les capacités du patient lors d'une réhabilitation pulmonaire. Par ailleurs, la probabilité de survenue d'une insuffisance cardiaque est élevée jusqu'à six mois après une exacerbation de BPCO [28].

L'évaluation aux urgences d'un patient BPCO admis pour dyspnée nécessite donc une appréciation fine des différents mécanismes liés à une éventuelle exacerbation de BPCO et/ ou une insuffisance cardiaque. C'est uniquement après cette appréciation méticuleuse qu'un traitement adapté, équilibré, pourra être mis en place. Le dosage des biomarqueurs cardiaques (BNP ou NT-pro-BNP) peut aider l'urgentiste dans cette démarche [18]. L'échographie pleuropulmonaire et cardiaque au lit du patient joue également un rôle primordial. Elle pourrait notamment permettre au médecin urgentiste d'avoir non pas une vision dichotomique (cœur vs poumon), mais d'apprécier la contribution relative des insuffisances cardiaque et ventilatoire chez le patient BPCO. Sa réalisation peut cependant être difficile du fait de la distension thoracique. L'administration de diurétiques devra être prudente en cas d'insuffisance cardiaque associée pour ne pas majorer les troubles de l'équilibre acidobasique [26,27]. L'utilisation du furosémide se fera toujours avec une surveillance étroite de la kaliémie, une hypokaliémie pouvant participer à l'épuisement des muscles respiratoires.

\section{Ischémie myocardique et cérébrale}

Le risque d'accident vasculaire cérébral (AVC) est multiplié par un facteur 1,2 à 1,7 après l'exacerbation [29-31], ce surrisque étant observé jusqu'à sept semaines après l'épisode. Il apparaît par contre moins élevé chez les patients présentant plus de deux exacerbations par an, illustrant le concept de phénotypes différents parmi cette population [9,32-34]. Plusieurs auteurs ont en effet décrit des populations différentes de patients BPCO, définies par des caractéristiques particulières (par exemple : une fonction ventilatoire peu altérée et de nombreuses comorbidités) et associées à une évolution spécifique de leur maladie (par exemple stabilité de la fonction respiratoire et survenue d'infarctus du myocarde [IDM]) $[32,33]$. L'incidence de l'IDM dans les suites immédiates d'une exacerbation est, elle, augmentée d'un facteur 2 à 10 $[27,28,30,35]$, et ce risque semble significatif jusqu'au $30^{\mathrm{e}}$ jour après l'exacerbation. La concentration sanguine des troponines est un marqueur indépendant du devenir des patients BPCO en exacerbation. Ainsi, de 10 à $70 \%$ des patients présentent des concentrations élevées de troponines associées à une surmortalité à moyen terme extrêmement importante [36-38]. Une augmentation de la troponine est associée à un risque accru de mortalité observé à 30 jours (odds ratio $[\mathrm{OR}]: 6,3$; intervalle de confiance à $95 \%$ [IC $95 \%$ ] :2,4-16,5) [36]. Dans une méta-analyse récente, l'élé- vation de la troponine était significativement liée à un risque accru de mortalité toutes causes confondues (OR : 1,69 ; IC $95 \%: 1,25-2,29)$ [39].

La physiopathologie de la BPCO, et plus particulièrement de l'exacerbation, explique les surrisques observés. Bien entendu, il existe des facteurs de risque communs à la BPCO et aux AVC et IDM : tabac, hypertension, diabète, etc. Mais certains mécanismes physiologiques sont plus particulièrement impliqués dans ces phénomènes ischémiques. L'inflammation systémique et locale est l'un d'eux. Elle provoque l'activation plaquettaire ainsi qu'une coagulation microvasculaire $[40,41]$. Elle induit le recrutement de médiateurs et de cellules pro-inflammatoires qui altèrent les membranes vasculaires [42]. L'hypoxie et le stress oxydant sont également impliqués dans la survenue d'événements ischémiques au décours de la maladie BPCO [42]. Enfin, l'augmentation de la consommation en oxygène du myocarde, liée soit à l'exacerbation elle-même, soit aux traitements initiés, augmente le risque d'infarctus de type 2 (absence de thrombose coronaire, mais déséquilibre entre apport et besoin en oxygène).

Le diagnostic d'événements ischémiques lors d'une exacerbation de BPCO peut être difficile. Bien entendu, tout patient BPCO présentant une exacerbation bénéficie, à l'admission, d'un électrocardiogramme qui permettra de faire le diagnostic d'un syndrome coronarien avec élévation du segment ST. La réalisation d'un électrocardiogramme est répétée dans le temps lors de l'hospitalisation, surtout en cas de douleur thoracique. Lorsque la recherche d'un infarctus du myocarde est systématique en période d'exacerbation, $25 \%$ des patients présentent des scores CIIS (Cardiac Infarction Injury Score) élevés [43]. Seules $30 \%$ de ces souffrances sont effectivement reconnues en pratique [43]. Le dosage des troponines a vraisemblablement un intérêt, notamment si les troponines sont indosables, ce qui pourrait indiquer un faible risque d'événement cardiovasculaire (valeur prédictive négative) [39]. Concernant une éventuelle prévention de ces risques ischémiques, il n'existe pour l'heure que très peu de données scientifiques fiables. Il semblerait que la prise d'aspirine au long cours réduise le risque de survenue d'AVC et qu'elle soit associée à une mortalité moindre jusqu'à un an après l'exacerbation [31,44]. Néanmoins, l'instauration d'un traitement antiagrégant au cours d'une exacerbation de BPCO ne peut être recommandée aujourd'hui. Notre rôle d'urgentiste est au minimum de maintenir toute prescription habituelle d'antithrombotiques au cours de l'hospitalisation ( $50 \%$ des patients admis pour exacerbation de BPCO sont sous antithrombotiques au long cours [12]).

\section{Maladie thromboembolique}

La prévalence de la maladie thromboembolique est élevée chez les patients BPCO en exacerbation $[45,46]$. La 
prévalence de l'embolie pulmonaire est d'environ $16 \%$ dans les exacerbations sans étiologie évidente [46]. Aux urgences, la prévalence de l'embolie pulmonaire chez les patients en exacerbation est probablement plus faible, de l'ordre de $5 \%$ [47]. Le risque de récurrence de thrombose veineuse profonde n'est pas plus élevé chez les patients BPCO, et la mortalité associée à une thrombose veineuse profonde n'est pas plus élevée dans cette population spécifique [48]. Au contraire, la survenue d'une embolie pulmonaire chez un patient BPCO semble associée à une surmortalité [49-51].

Sur le plan physiopathologique, l'association de la maladie thromboembolique avec la BPCO s'explique par différents facteurs. Premièrement, l'inflammation systémique qui caractérise la BPCO provoque une dysfonction endothéliale et une activation plaquettaire [52]. Deuxièmement, l'inflammation et l'hypoxie tissulaire provoquent une activation directe de la coagulation [53,54]. Enfin, il existe une stase veineuse du fait des modifications de pressions intrathoraciques, de la modification de la vasomotricité veineuse et de l'immobilisation des patients en exacerbation. Tous ces phénomènes concourent au développement de la maladie thromboembolique chez les patients en phase stable mais surtout en exacerbation de BPCO.

D'un point de vue clinique, l'association maladie thromboembolique et BPCO se traduit par une surmortalité [49-51]. Il est donc primordial pour les médecins d'identifier les situations à risque (immobilisation du fait de la dyspnée) et de diagnostiquer précocement tout événement thromboembolique. Notamment, le diagnostic doit être systématiquement évoqué lors d'une exacerbation avec douleur thoracique inexpliquée, en cas d'hyperventilation alvéolaire ou d'hypoxémie majeure en l'absence de pathologie infectieuse bronchopulmonaire [46]. Une embolie pulmonaire doit être suspectée devant toute exacerbation d'étiologie non évidente. L'utilisation des algorithmes traditionnels de diagnostic de maladie thromboembolique est recommandée. La valeur limite des D-dimères chez les patients BPCO est vraisemblablement plus élevée, mais il n'existe pas, à l'heure actuelle, d'algorithme décisionnel spécifique au patient BPCO [55]. Enfin, il n'existe pas de preuve formelle de l'intérêt d'une anticoagulation préventive lors d'une exacerbation de BPCO. À l'heure actuelle, seules des données préliminaires laissent à penser que l'anticoagulation préventive du patient BPCO hospitalisé et alité aurait un intérêt, a fortiori, s'il a des signes d'insuffisance cardiaque droite $[56,57]$.

\section{Insuffisance rénale}

La prévalence de l'insuffisance rénale chronique chez les patients BPCO est estimée entre 10 et $20 \%$ [58,59]. Elle augmente avec l'âge et est plus fréquente chez les patients dénutris [59]. La présence d'une insuffisance rénale chro- nique est associée à une plus haute probabilité d'exacerbation et à une surmortalité à un an [60-62]. La prévalence d'une insuffisance rénale aiguë au cours d'une exacerbation est d'environ $20 \%$ et est associée à une surmortalité [63,64]. La probabilité de ventilation mécanique et d'admission en réanimation est plus élevée chez les patients BPCO en exacerbation présentant une insuffisance rénale aiguë [64]. Le dosage de la créatinémie semble avoir un intérêt limité pour stratifier le risque des patients BPCO, notamment chez les patients âgés, fragiles et/ou dénutris [58].

Les mécanismes intriqués de la BPCO et de l'insuffisance rénale sont nombreux. Bien entendu, des comorbidités communes existent : diabète, hypertension artérielle, insuffisance cardiaque. L'inflammation induite par une exacerbation de BPCO est encore ici au centre des interactions physiopathologiques : microthrombi intravasculaires, dysfonction endothéliale, activation sympathique, anomalies de rigidité artérielle sont autant de facteurs précipitant la fonction rénale [58]. La présence d'une insuffisance cardiaque chronique peut augmenter le risque d'insuffisance rénale du fait d'un potentiel bas débit sanguin rénal et de l'utilisation de traitements à retentissement rénal. Enfin, les reins et les poumons constituent les deux organes majeurs de la régulation de la balance acidobasique de l'organisme. En cas d'insuffisance rénale, la présence de troubles mixtes acidobasiques lors des exacerbations des BPCO est augmentée. Ces troubles mixtes peuvent rendre complexe la prise en charge des patients et sont associés à une augmentation de la probabilité de ventilation mécanique et d'admission en réanimation [27].

\section{Douleur}

La douleur est un symptôme fréquent chez les patients BPCO, même à des stades non palliatifs de la maladie [6567]. De 21 à $72 \%$ des patients présentent une douleur à la phase stable de leur maladie. Une méta-analyse suggère que la douleur est davantage prévalente chez les patients avec une BPCO modérée que chez les patients atteints de BPCO sévère [68]. La douleur a un impact majeur sur la qualité de vie, car elle contribue à la fois à la réduction de l'activité physique et au développement de troubles de l'humeur $[69,70]$. Les patients BPCO douloureux participent moins au programme de réhabilitation pulmonaire [71]. Elle serait également un facteur de non-observance des patients [72]. Lors des exacerbations, la douleur pourrait participer à l'inconfort des patients et favoriser l'échec des thérapeutiques, au premier rang desquelles la ventilation non invasive.

Les mécanismes sous-jacents de la douleur chez les patients avec la BPCO ne sont pas clairement identifiés, bien que les troubles musculosquelettiques, les comorbidités et l'inflammation systémique aient tous été suggérés comme facteurs contributifs ainsi que l'augmentation du travail et 
de l'activité des muscles respiratoires au cours de la respiration $[68,72]$. Une étude a montré que la dyspnée, mais non la fonction pulmonaire, était associée à la douleur [73]. Toutefois, à ce jour, peu d'études ont abordé la question de savoir si la douleur augmentait pendant les exacerbations aiguës de la BPCO. Notre équipe a montré que plus de $90 \%$ des patients en exacerbation présentent une douleur significative [74]. Cette douleur est principalement localisée au thorax et à la tête [74]. De façon intéressante, l'intensité douloureuse était principalement associée à l'anxiété des patients dans cette étude. L'anxiété est un symptôme fréquent chez les patients BPCO, et elle est très étroitement associée à la mortalité [75]. Anxiété, douleur et dyspnée sont des symptômes intriqués chez le patient BPCO.

Le traitement antalgique des patients BPCO hors phase palliative est difficile. Bien entendu, le paracétamol est la molécule de première intention du fait de son profil de sécurité. Néanmoins, l'intensité douloureuse est bien souvent modérée à intense, et les antalgiques faibles sont alors insuffisants. Les antalgiques plus forts sont souvent des dérivés opioïdes faibles ou forts, avec possiblement des effets indésirables en termes d'hypoventilation centrale. L'utilisation de la morphine par voie orale chez les patients BPCO non palliatifs et hors exacerbation est discutée. Les résultats de la littérature sont en effet divergents, et les études de qualité médiocres [76,77]. Il n'existe aucune donnée sur l'antalgie du patient BPCO en phase d'exacerbation.

\section{Quelle évaluation globale du patient en exacerbation de BPCO ?}

La BPCO est donc une maladie systémique, et l'évaluation des patients BPCO en exacerbation est multidimensionnelle $[78,79]$. Certains scores de sévérité des exacerbations de BPCO commencent à prendre en compte les aspects extrapulmonaires de cette maladie. Ainsi, le score DECAF pour Dyspnea, Eosinopenia, Consolidation, Acidemia and atrial Fibrillation stratifie le risque de mortalité à un mois en fonction de marqueurs de la ventilation, de l'inflammation et du risque cardiovasculaire des patients [80]. En pratique, un médecin urgentiste prenant en charge un patient BPCO en exacerbation effectue une évaluation globale du patient qui repose bien entendu sur l'appréciation de l'état respiratoire du patient mais aussi sur les dimensions cardiovasculaire, embolique (non abordée dans cette revue), rénale et du confort du patient. Cette évaluation est clinique et peut s'aider d'outils d'imagerie et de biologie (Tableau 2). De nombreuses études cliniques sont encore nécessaires avant de proposer un algorithme fiable de prise en charge globale du patient en exacerbation de BPCO.
Tableau 2 Proposition d'évaluation simple des aspects extrapulmonaires lors de l'admission d'un patient pour exacerbation de bronchopneumopathie chronique obstructive

\section{Existe-t-il un risque cardiovasculaire accru ?}

Recherche d'antécédents et de comorbidités cardiovasculaires

Présence d'un traitement habituel par antiagrégant

plaquettaire

Électrocardiogramme et échographie cardiaque

et pleuropulmonaire

Dosage des Nt-pro-BNP, des BNP et des troponines

à l'admission

2. Existe-t-il un risque thromboembolique accru ?

Recherche d'antécédents de maladie thromboembolique

Identification de facteurs de risque (alitement, foyer

de pneumopathie, etc.)

Présence d'un traitement habituel par antithrombotiques

3. Quelle est la fonction rénale de mon patient ?

Recherche d'antécédents d'insuffisance rénale

Appréciation de l'état d'hydratation

Dosage de la concentration plasmatique de créatinine

4. Le patient est-il confortable ?

Évaluation de la douleur (échelle numérique)

Évaluation de l'anxiété

Recherche d'un syndrome dépressif chronique

5. Balance bénéfice/risque des traitements ?

Notamment : anticholinergiques et diurétiques

\section{Conclusion}

La BPCO est une maladie systémique. L'évaluation et le traitement des patients en exacerbation de $\mathrm{BPCO}$ requièrent donc une évaluation de l'état ventilatoire du patient mais aussi des aspects extrapulmonaires de cette maladie, au premier rang desquels les pathologies cardiovasculaires. Cette évaluation médicale globale permet d'adapter au mieux les thérapeutiques et le parcours de soins selon le phénotype du patient et de son exacerbation. Il n'existe cependant pas de recommandation formelle intégrant les aspects multisystémiques de la BPCO dans la pratique clinique des urgentistes, car les preuves scientifiques restent à venir.

Liens d'intérêts : Damien Viglino a reçu des fonds de recherche d'AstraZeneca. Maxime Maignan a reçu des fonds de recherche d'Agir@Dom, Mundipharma, Biomérieux, Roche Diagnotics.

\section{Références}

1. Almagro P, Cabrera FJ, Diez J, et al (2012) Comorbidities and short-term prognosis in patients hospitalized for acute exacerbation 
of COPD: the EPOC en Servicios de medicina interna (ESMI) study. Chest 142:1126-33

2. Gale NS, Bolton CE, Duckers JM, et al (2012) Systemic comorbidities in bronchiectasis. Chron Respir Dis 9:231-8

3. Maclay JD, MacNee W (2013) Cardiovascular disease in COPD: mechanisms. Chest 143:798-807

4. Vivodtzev I, Tamisier R, Baguet JP, et al (2014) Arterial stiffness in COPD. Chest 145:861-75

5. Agusti A, Calverley PMA, Celli B, et al (2010) Characterisation of COPD heterogeneity in the ECLIPSE cohort. Respir Res $11: 122$

6. Wang Y, Stavem K, Dahl FA, et al (2014) Factors associated with a prolonged length of stay after acute exacerbation of chronic obstructive pulmonary disease (AECOPD). Int J Chron Obstruct Pulmon Dis 9:99-105

7. Perera PN, Armstrong EP, Sherrill DL, Skrepnek GH (2012) Acute exacerbations of COPD in the United States: inpatient burden and predictors of costs and mortality. COPD 9:131-41

8. Wang Y, Stavem K, Dahl FA, et al (2014) Factors associated with a prolonged length of stay after acute exacerbation of chronic obstructive pulmonary disease (AECOPD). Int J Chron Obstruct Pulmon Dis 9:99-105

9. Garcia-Aymerich J, Gómez FP, Benet M, et al (2011) Identification and prospective validation of clinically relevant chronic obstructive pulmonary disease (COPD) subtypes. Thorax 66:430-7

10. Burgel PR, Paillasseur JL, Peene B, et al (2012) Two distinct chronic obstructive pulmonary disease (COPD) phenotypes are associated with high risk of mortality. PLoS ONE 7:e51048

11. Malerba M, Clini E, Malagola M, Avanzi GC (2013) Platelet activation as a novel mechanism of atherothrombotic risk in chronic obstructive pulmonary disease. Expert Rev Hematol 6:475-83

12. Harrison MT, Short P, Williamson PA, et al (2014) Thrombocytosis is associated with increased short and long term mortality after exacerbation of chronic obstructive pulmonary disease: a role for antiplatelet therapy? Thorax 69:609-15

13. Macchia A, Rodriguez Moncalvo JJ, Kleinert M, et al (2012) Unrecognised ventricular dysfunction in COPD. Eur Respir $\mathbf{J}$ 39:51-8

14. Boudestein LCM, Rutten FH, Cramer MJ, et al (2009) The impact of concurrent heart failure on prognosis in patients with chronic obstructive pulmonary disease. Eur J Heart Fail 11:11828

15. Huiart L, Ernst P, Suissa S (2005) Cardiovascular morbidity and mortality in COPD. Chest 128:2640-6

16. Fuso L, Incalzi RA, Pistelli R, et al (1995) Predicting mortality of patients hospitalized for acutely exacerbated chronic obstructive pulmonary disease. Am J Med 98:272-7

17. Høiseth AD, Omland T, Karlsson BD, et al (2013) Standardized evaluation of lung congestion during COPD exacerbation better identifies patients at risk of dying. Int $\mathrm{J}$ Chron Obstruct Pulmon Dis 8:621-9

18. Høiseth AD, Omland T, Hagve TA, et al (2012) NT-proBNP independently predicts long term mortality after acute exacerbation of COPD - a prospective cohort study. Respir Res 13:97

19. Kalhan R, Mutharasan RK (2018) Reducing readmissions in patients with both heart failure and COPD. Chest 154:1230-8

20. Orea-Tejeda A, Navarrete-Peñaloza AG, Verdeja-Vendrell L, et al (2018) Right heart failure as a risk factor for severe exacerbation in patients with chronic obstructive pulmonary disease: prospective cohort study. Clin Respir J 12:2635-41

21. Baldwin ED, Cournand A, Richards DW (1949) Pulmonary insufficiency; a study of 122 cases of chronic pulmonary emphysema. Medicine 28:201-37

22. Neder JA, Rocha A, Alencar MCN, et al (2018) Current challenges in managing comorbid heart failure and COPD. Expert Rev Cardiovasc Ther 16:653-73
23. Wells JM, Dransfield MT (2013) Pathophysiology and clinical implications of pulmonary arterial enlargement in COPD. Int $\mathrm{J}$ Chron Obstruct Pulmon Dis 8:509-21

24. Thomson AJ, Drummond GB, Waring WS, et al (2006) Effects of short-term isocapnic hyperoxia and hypoxia on cardiovascular function. J Appl Physiol 101:809-16

25. Horodinschi RN, Bratu OG, Dediu GN, et al (2019) Heart failure and chronic obstructive pulmonary disease: a review. Acta Cardiol 16:1-8

26. Schiavo A, Renis M, Polverino M, et al (2016) Acid-base balance, serum electrolytes and need for non-invasive ventilation in patients with hypercapnic acute exacerbation of chronic obstructive pulmonary disease admitted to an internal medicine ward. Multidiscip Respir Med 11:23

27. Terzano C, Di Stefano F, Conti V, et al (2012) Mixed acid-base disorders, hydroelectrolyte imbalance and lactate production in hypercapnic respiratory failure: the role of noninvasive ventilation. PLoS ONE 7:e35245

28. Halpin DMG, Decramer M, Celli B, et al (2011) Risk of nonlower respiratory serious adverse events following COPD exacerbations in the 4-year UPLIFT ${ }^{\mathbb{R}}$ trial. Lung 189:261-8

29. Donaldson GC, Hurst JR, Smith CJ, et al (2010) Increased risk of myocardial infarction and stroke following exacerbation of COPD. Chest 137:1091-7

30. Portegies MLP, Lahousse L, Joos GF, et al (2016) Chronic obstructive pulmonary disease and the risk of stroke. The Rotterdam study. Am J Respir Crit Care Med 193:251-8

31. Rothnie KJ, Connell O, Müllerová H, et al (2018) Myocardial infarction and ischemic stroke after exacerbations of chronic obstructive pulmonary disease. Ann Am Thorac Soc 15:935-46

32. Agusti A, Calverley PMA, Celli B, et al (2010) Characterisation of COPD heterogeneity in the ECLIPSE cohort. Respir Res $11: 122$

33. Han MK, Agusti A, Calverley PM, et al (2010) Chronic obstructive pulmonary disease phenotypes: the future of COPD. Am J Respir Crit Care Med 182:598-604

34. Burgel PR, Paillasseur JL, Caillaud D, et al (2010) Clinical COPD phenotypes: a novel approach using principal component and cluster analyses. Eur Respir J 36:531-9

35. Rothnie KJ, Yan R, Smeeth L, Quint JK (2015) Risk of myocardial infarction (MI) and death following MI in people with chronic obstructive pulmonary disease (COPD): a systematic review and meta-analysis. BMJ Open 5:e007824

36. Chang CL, Robinson SC, Mills GD, et al (2011) Biochemical markers of cardiac dysfunction predict mortality in acute exacerbations of COPD. Thorax 66:764-8

37. Høiseth AD, Neukamm A, Karlsson BD, et al (2011) Elevated high-sensitivity cardiac troponin $\mathrm{T}$ is associated with increased mortality after acute exacerbation of chronic obstructive pulmonary disease. Thorax 66:775-81

38. Baillard C, Boussarsar M, Fosse JP, et al (2003) Cardiac troponin I in patients with severe exacerbation of chronic obstructive pulmonary disease. Intensive Care Med 29:584-9

39. Pavasini R, d'Ascenzo F, Campo G, et al (2015) Cardiac troponin elevation predicts all-cause mortality in patients with acute exacerbation of chronic obstructive pulmonary disease: systematic review and meta-analysis. Int J Cardiol 191:187-93

40. Polosa R, Malerba M, Cacciola RR, et al (2013) Effect of acute exacerbations on circulating endothelial, clotting and fibrinolytic markers in COPD patients. Intern Emerg Med 8:567-74

41. Sabit R, Thomas P, Shale DJ, et al (2010) The effects of hypoxia on markers of coagulation and systemic inflammation in patients with COPD. Chest 138:47-51

42. Corlateanu A, Covantev S, Mathioudakis AG, et al (2018) Chronic obstructive pulmonary disease and stroke. COPD 15:405-13 
43. Brekke PH, Omland T, Smith P, Søyseth V (2008) Underdiagnosis of myocardial infarction in COPD - Cardiac Infarction Injury Score (CIIS) in patients hospitalised for COPD exacerbation. Respir Med 102:1243-7

44. Pavasini R, Biscaglia S, d'Ascenzo F, et al (2016) Antiplatelet treatment reduces all-cause mortality in COPD patients: a systematic review and meta-analysis. COPD 13:509-14

45. Gunen H, Gulbas G, In E, et al (2010) Venous thromboemboli and exacerbations of COPD. Eur Respir J 35:1243-8

46. Aleva FE, Voets LWLM, Simons SO, et al (2017) Prevalence and localization of pulmonary embolism in unexplained acute exacerbations of COPD: a systematic review and meta-analysis. Chest 151:544-54

47. Rizkallah J, Man SFP, Sin DD (2009) Prevalence of pulmonary embolism in acute exacerbations of COPD: a systematic review and metaanalysis. Chest 135:786-93

48. Le Mao R, Tromeur C, Bazire A, et al (2017) Risk of recurrent venous thromboembolism in COPD patients: results from a prospective cohort study. Eur Respir J 50(1):1700094

49. Carson JL, Terrin ML, Duff A, Kelley MA (1996) Pulmonary embolism and mortality in patients with COPD. Chest 110:1212-9

50. Bertoletti L, Quenet S, Mismetti P, et al (2012) Clinical presentation and outcome of venous thromboembolism in COPD. Eur Respir J 39:862-8

51. Bertoletti L, Quenet S, Laporte S, et al (2013) Pulmonary embolism and 3-month outcomes in 4,036 patients with venous thromboembolism and chronic obstructive pulmonary disease: data from the RIETE registry. Respir Res 14:75

52. Malerba M, Nardin M, Radaeli A, et al (2017) The potential role of endothelial dysfunction and platelet activation in the development of thrombotic risk in COPD patients. Expert Rev Hematol $10: 821-32$

53. Sabit R, Thomas P, Shale DJ, et al (2010) The effects of hypoxia on markers of coagulation and systemic inflammation in patients with COPD. Chest 138:47-51

54. Undas A, Jankowski M, Kaczmarek P, et al (2011) Thrombin generation in chronic obstructive pulmonary disease: dependence on plasma factor composition. Thromb Res 128:e24-e8

55. Akpinar EE, Hoşgün D, Doğanay B, et al (2013) Should the cutoff value of D-dimer be elevated to exclude pulmonary embolism in acute exacerbation of COPD? J Thorac Dis 5:430-4

56. Shi X, Li H (2013) Anticoagulation therapy in patients with chronic obstructive pulmonary disease in the acute exacerbation stage. Exp Ther Med 5:1367-70

57. Andersson C, Hansen PW, Steffensen IE, et al (2019) Mortality associated with cardiovascular drugs in patients with chronic obstructive pulmonary disease and right-sided heart failure - a danish nationwide registry-based study. Eur J Intern Med 63:56-61

58. Incalzi RA, Corsonello A, Pedone C, et al (2010) Chronic renal failure: a neglected comorbidity of COPD. Chest 137:831-7

59. Gjerde B, Bakke PS, Ueland T, et al (2012) The prevalence of undiagnosed renal failure in a cohort of COPD patients in western Norway. Respir Med 106:361-6

60. Terzano C, Colamesta V, Unim B, et al (2017) Chronic obstructive pulmonary disease (COPD) exacerbation: impact of comorbidities on length and costs during hospitalization. Eur Rev Med Pharmacol Sci 21:3680-9

61. Singanayagam A, Schembri S, Chalmers JD (2013) Predictors of mortality in hospitalized adults with acute exacerbation of chronic obstructive pulmonary disease. Ann Am Thorac Soc 10:81-9
62. Fedeli U, De Giorgi A, Gennaro N, et al (2017) Lung and kidney: a dangerous liaison? A population-based cohort study in COPD patients in Italy. Int J Chron Obstruct Pulmon Dis 12:443-50

63. Barakat MF, McDonald HI, Collier TJ, et al (2015) Acute kidney injury in stable COPD and at exacerbation. Int J Chron Obstruct Pulmon Dis 10:2067-77

64. Cao CC, Chen DW, Li J, et al (2018) Community-acquired versus hospital-acquired acute kidney injury in patients with acute exacerbation of COPD requiring hospitalization in China. Int $\mathrm{J}$ Chron Obstruct Pulmon Dis 13:2183-90

65. HajGhanbari B, Holsti L, Road JD, Darlene Reid W (2012) Pain in people with chronic obstructive pulmonary disease (COPD). Respir Med 106:998-1005

66. HajGhanbari B, Garland SJ, Road JD, Reid WD (2013) Pain and physical performance in people with COPD. Respir Med 107:1692-9

67. Bentsen SB, Rustøen T, Miaskowski C (2011) Prevalence and characteristics of pain in patients with chronic obstructive pulmonary disease compared to the Norwegian general population. J Pain 12:539-45

68. van Dam van Isselt EF, Groenewegen-Sipkema KH, Spruitvan Eijk M, et al (2014) Pain in patients with COPD: a systematic review and meta-analysis. BMJ Open 4:e005898

69. Borge CR, Wahl AK, Moum T (2011) Pain and quality of life with chronic obstructive pulmonary disease. Heart Lung 40: e90-e101

70. Lee AL, Harrison SL, Goldstein RS, Brooks D (2018) An exploration of pain experiences and their meaning in people with chronic obstructive pulmonary disease. Physiother Theory Pract 34:765-72

71. Harrison SL, Lee AL, Elliott-Button HL, et al (2017) The role of pain in pulmonary rehabilitation: a qualitative study. Int J Chron Obstruct Pulmon Dis 12:3289-99

72. Janssen DJA, de Hosson SM, Bij de Vaate E, et al (2015) Attitudes toward opioids for refractory dyspnea in COPD among Dutch chest physicians. Chron Respir Dis 12:85-92

73. Schön D, Rosenkranz M, Regelsberger J, et al (2008) Reduced perception of dyspnea and pain after right insular cortex lesions. Am J Respir Crit Care Med 178:1173-9

74. Maignan M, Chauny JM, Daoust R, et al (2019) Pain during exacerbation of chronic obstructive pulmonary disease: a prospective cohort study. PLoS ONE doi.org/10.1371/journal.pone.0217370

75. Divo M, Cote C, de Torres JP, et al (2012) Comorbidities and risk of mortality in patients with chronic obstructive pulmonary disease. Am J Respir Crit Care Med 186:155-61

76. Vozoris NT, Wang X, Fischer HD, et al (2016) Incident opioid drug use and adverse respiratory outcomes among older adults with COPD. Eur Respir J 48:683-93

77. Viglino D, Maignan M (2016) Opioids and adverse outcomes in elderly chronic obstructive pulmonary disease patients. Eur Respir J 48:1817

78. Euceda G, Kong WT, Kapoor A, et al (2018) The effects of a comprehensive care management program on readmission rates after acute exacerbation of COPD at a community-based academic hospital. Chronic Obstr Pulm Dis 5:185-92

79. Lane ND, Brewin K, Hartley TM, et al (2018) Specialist emergency care and COPD outcomes. BMJ Open Respir Res 5: e000334

80. Echevarria C, Steer J, Heslop-Marshall K, et al (2016) Validation of the DECAF score to predict hospital mortality in acute exacerbations of COPD. Thorax 71:133-40 